\title{
Acid Treatment Halloysite Nanoclay: Eco-Friendly Heterogeneous Catalyst for the Synthesis of Pyrrole Derivatives $^{\dagger}$
}

\author{
Ali Maleki * and Zoleikha Hajizadeh \\ Catalysts and Organic Synthesis Research Laboratory, Department of Chemistry, Iran University of Science \\ and Technology, Tehran 16846-13114, Iran; hajizadeh.zoleikha@gmail.com \\ * Correspondence: maleki@iust.ac.ir \\ + Presented at the 22nd International Electronic Conference on Synthetic Organic Chemistry, 15 November- \\ 15 December 2018; Available Online: https://sciforum.net/conference/ecsoc-22.
}

Published: 14 November 2018

\begin{abstract}
In this study, halloysite nanotubes (HNTs) as a green and available nano-mineral were used. The hydroxyl groups of HNTs were increased by acid treatment. So, the efficiency of HNTs was improved. The catalyst application of acid treatment HNTs was investigated in the synthesis of pyrrole as an important heterocycle compound. The catalyst was characterized by Fourier transforms infrared spectroscopy (FT-IR). Easy and simple method, high efficiency and eco-friendly are the advantages of this report.
\end{abstract}

Keywords: halloysite nanotubes; acid treatment; nanocatalyst; pyrrole

\section{Introduction}

Halloysite $\left(\mathrm{Al}_{2} \mathrm{Si}_{2} \mathrm{O}_{5}(\mathrm{OH})_{4} \cdot \mathrm{nH}_{2} \mathrm{O}\right)$ is a subset of aluminosilicate minerals. Halloysite nanotubes (HNTs) were mined from natural deposits in countries like China, New Zealand, America, Brazil, and France. Nanotube structure, availability, eco-friendliness, and porosity are very important features for HNTs [1]. HNTs are used as a catalyst application in different reaction [2,3]. The external and internal surfaces of HNTs are composed of silicon-oxygen tetrahedron and alumina oxygen octahedrons, respectively. The reaction of acid with both the outer and inner surfaces of the nanotubes cause the $\mathrm{AlO}_{6}$ octahedral layers to dissolve and the occurance of dealumination [4]. The acid treatment of halloysite is used as a traditional chemical activation method for improving the performance of its catalytic activity. Nowadays, nanocatalysts have attracted much attention as a beneficial strategy in green chemistry [5].

Nitrogen-based heterocyclic compounds have been widely used in the pharmaceutical industry [6]. Therefore, pyrrole as a nitrogen-based heterocyclic with biological activities is more considerable by scientists [7]. Several methods and catalysts were reported for the synthesis of this important framework [8]. In continuation of our research [9-14] and due to the importance of pyrrole scaffold, herein, an efficient synthesis of pyrrole derivatives was carried out in the presence of a catalytic amount of acid treatment halloysite nanocatalyst in ethanol at room temperature in high yields. The solid nanocatalyst can be recovered easily and reused without any significant loss of the catalytic activity (Scheme 1). 


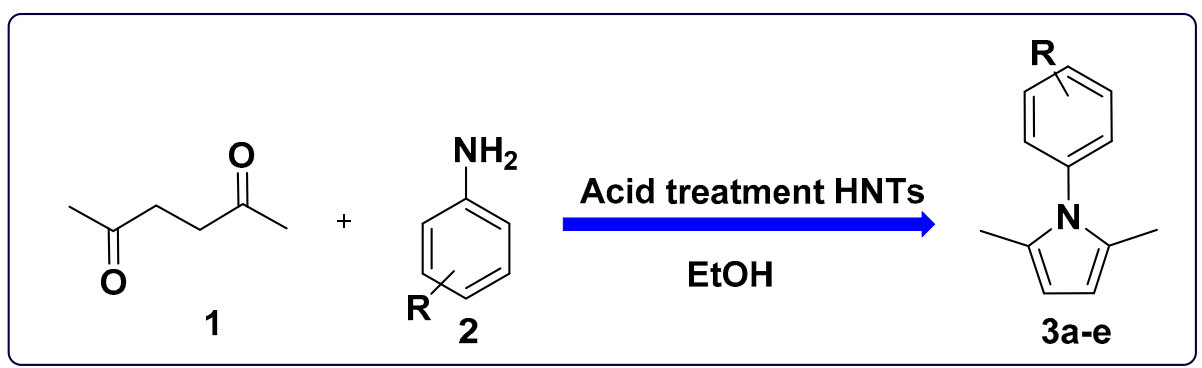

Scheme 1. The synthesis of pyrroles in the presence of halloysite nanoclay catalyst.

\section{Experimental}

\subsection{General}

All raw materials and solvents required were provided from Merck and Aldrich. The FT-IR spectrum of the product was taken by Shimadzu IR-470 spectrometer on KBr pellet. Melting points were measured with an Electrothermal 9100 apparatus and are uncorrected.

\subsection{Synthesis of Acid Treatment HNTs}

At first, $0.5 \mathrm{~g}$ of halloysite was added to a $10 \mathrm{~mL}$ deionized water. The mixture was stirred for $10 \mathrm{~min}$ and the uniform solution was achieved. Then, $50 \mathrm{~mL}$ of $\mathrm{HCl}$ solution $(3 \mathrm{M})$ was added to the halloysite solution, and the mixture was stirred for $3 \mathrm{~h}$. The nanoclay powder was washed with distilled water twice. Finally, the obtained nanocomposite was dried at $100{ }^{\circ} \mathrm{C}$ for $4 \mathrm{~h}$.

\subsection{General Procedure for the Synthesis of Pyrrole Derivatives $3 a-e$}

Acetonylacetone ( $1 \mathrm{mmol}, 0.114 \mathrm{~g})$, aniline $(1 \mathrm{mmol}, 0.093 \mathrm{~g})$, and catalyst $(0.06 \mathrm{~g})$ were mixed in ethanol. The mixture was stirred in an air atmosphere at room temperature for $1 \mathrm{~h}$. After the completion of the reaction, as indicated by TLC, the catalyst was removed easily by filtration. The crude product was filtered and recrystallized from ethanol to obtain products in good to high yields.

\section{Results and Discussion}

FT-IR spectroscopy was used to confirm the synthesis of acid treatment HNTs (Figure 1). The bands at 1035, 794, 792, and $692 \mathrm{~cm}^{-1}$ were attributed to the stretching vibrations of Si-O in both HNTs and acid treatment HNTs. As shown in the HNTs' spectrum, the peak at $912 \mathrm{~cm}^{-1}$ was assigned to the bending vibration of $\mathrm{Al}-\mathrm{OH}$, while, this peak was disappeared in acid treatment HNTs. Also, acid treatment increased the hydroxyl groups. The broad and intense band around $3000 \mathrm{~cm}^{-1} \mathrm{can} \mathrm{confirm}$ this result. 


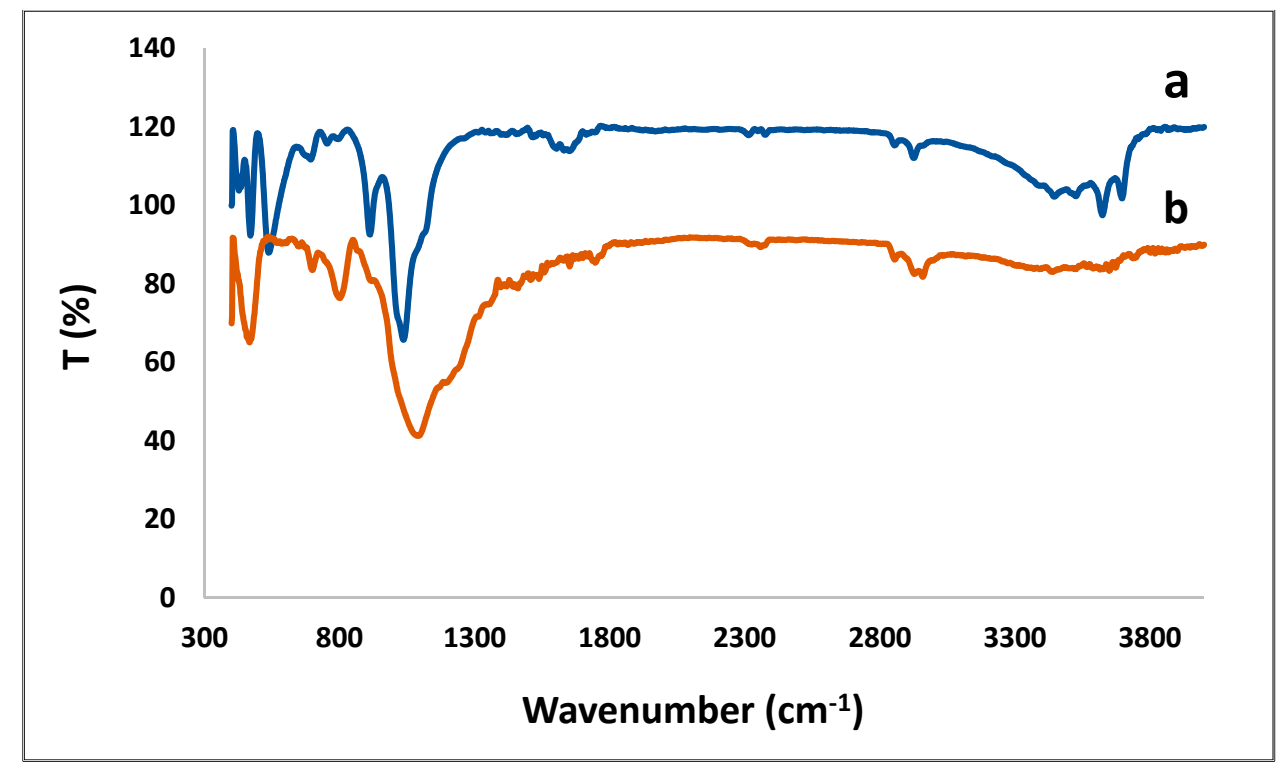

Figure 1. FT-IR spectra of: (a) HNTs and (b) acid treatment HNTs.

\section{Conclusions}

In summary, the green, eco-friendly, available, and efficient nanocatalyst base on HNTs nanoclay was introduced. The acid treatment HNTs with high performance was used in the synthesis of pyrrole derivatives. High yield, green media, and short reaction time are the benefits of this study. This research is under progress in our research laboratory.

Acknowledgments: The authors acknowledge the partial support from the Research Council of the Iran University of Science and Technology.

\section{References}

1. Liu, M.; Jia, Z.; Jia, D.; Zhou, C. Recent advance in research on halloysite nanotubes-polymer nanocomposite. Prog. Polym. Sci. 2014, 39, 1498-1525.

2. Maleki, A.; Hajizadeh, Z.; Firouzi-Haji, R. Eco-friendly functionalization of magnetic halloysite nanotube with SO3H for synthesis of dihydropyrimidinones. Microporous Mesoporous Mater. 2018, 259, 46-53.

3. Hajizadeh, Z.; Maleki, A. Poly (ethylene imine)-modified magnetic halloysite nanotubes: A novel, efficient and recyclable catalyst for the synthesis of dihydropyrano [2, 3-c] pyrazole derivatives. Mol. Catal. 2018, 460, 87-93.

4. Joo, Y.; Sim, J.H.; Jeon, Y.; Lee, S.U.; Sohn, D. Opening and blocking the inner-pores of halloysite. Chem. Commun. 2013, 49, 4519-4521.

5. Maleki, A.; Hajizadeh, Z.; Abbasi, H. Surface modification of graphene oxide by citric acid and its application as a heterogeneous nanocatalyst in organic condensation reaction. Carbon Lett. 2018, 27, 42-49.

6. Azzam, R.A.; Mohareb, R.M. Multicomponent reactions of acetoacetanilide derivatives with aromatic aldehydes and cyanomethylene reagents to produce $4 \mathrm{H}$-pyran and 1, 4-dihydropyridine derivatives with antitumor activities. Chem. Pharm. Bull. 2015, 63, 1055-1064.

7. van Leusen, A.M.; Siderius, H.; Hoogenboom, B.E.; Leusen, D. A new and simple synthesis of the pyrrole ring system from Michael acceptors and tosylmethylisocyanides. Tetrahedron Lett. 1972, 13, 5337-5340.

8. Roland, R.U.; Zeitler, K.; Müller, T.J.J. A Novel One-Pot Pyrrole Synthesis via a Coupling- IsomerizationStetter- Paal- Knorr Sequence. Org. Lett. 2001, 3, 3297-3300.

9. Maleki, A. $\mathrm{Fe}_{3} \mathrm{O}_{4} / \mathrm{SiO}_{2}$ nanoparticles: An efficient and magnetically recoverable nanocatalyst for the onepot multicomponent synthesis of diazepines. Tetrahedron 2012, 68, 7827-7833.

10. Maleki, A. One-pot multicomponent synthesis of diazepine derivatives using terminal alkynes in the presence of silica-supported superparamagnetic iron oxide nanoparticles. Tetrahedron Lett. 2013, 54, 2055-2059.

11. Maleki, A. One-pot three-component synthesis of pyrido[2', $\left.1^{\prime}: 2,3\right]$ imidazo[4,5-c]isoquinolines using $\mathrm{Fe}_{3} \mathrm{O}_{4} @ \mathrm{SiO}_{2}-\mathrm{OSO}_{3} \mathrm{H}$ as an efficient heterogeneous nanocatalyst. RSC Adv. 2014, 4, 64169-64173. 
12. Maleki, A. Green oxidation protocol: Selective conversions of alcohols and alkenes to aldehydes, ketones and epoxides by using a new multiwall carbon nanotube-based hybrid nanocatalyst via ultrasound irradiation. Ultrason. Sonochem. 2018, 40, 460-464.

13. Maleki, A.; Ghassemi, M.; Firouzi-Haji, R. Green multicomponent synthesis of four different classes of sixmembered N-containing and O-containing heterocycles catalyzed by an efficient chitosan-based magnetic bionanocomposite. Pure Appl. Chem. 2018, 90, 387-394.

14. Maleki, A.; Ghalavand, R.; Firouzi-Haji, R. A novel and eco-friendly o-phenylendiamine stabilized on silica-coated magnetic nanocatalyst for the synthesis of indenoquinoline derivatives under ultrasonic assisted solvent-free conditions. Iran. J. Catal. 2018, 8, 221-229.

(C) 2019 by the authors. Licensee MDPI, Basel, Switzerland. This article is an open access article distributed under the terms and conditions of the Creative Commons Attribution (CC BY) license (http://creativecommons.org/licenses/by/4.0/). 